\title{
Intelligent techniques and architectures for autonomic clouds: introduction to the itaac special issue
}

\author{
Nick Antonopoulos ${ }^{1 *}$, Ashiq Anjum and Lee Gillam²
}

\author{
* Correspondence: N \\ Antonopoulos@derby.ac.uk \\ ${ }^{1}$ School of Computing and \\ Mathematics, Faculty of Business, \\ Computing and Law, University of \\ Derby, Kedleston Road, Derby DE22 \\ $1 \mathrm{~GB}$, UK \\ Full list of author information is \\ available at the end of the article
}

\begin{abstract}
Autonomic Computing introduces autonomy in software systems by making them capable of self- management and self-healing. Autonomic Clouds have emerged as a result of the application of autonomic techniques to cloud computing, resulting in robust, fault tolerant and resilient cloud architectures. The international workshop on Intelligent Techniques and Architectures for Autonomic Clouds (ITAAC 2011) was held in association with the 4th IEEE/ACM International Conference on Utility and Cloud Computing (UCC 2011)in Melbourne Australia to bring together researchers and practitioners across Cloud Computing, Intelligent Systems, and Autonomic Computing to discuss issues that drive the concepts, architectures and applications of autonomic clouds. This article provides background to the workshop and presents a brief summary of the papers that were accepted.
\end{abstract}

\section{Introduction}

Autonomic computing refers to principles and techniques for designing, building, deploying and managing computing systems with minimal human involvement. Autonomic computing makes systems adaptive by providing methods and means to enhance aspects of system autonomy, self-management, self-tuning, self-configuration, self-diagnosis, and self-healing.

Autonomic Clouds have emerged as a result of the application of autonomic techniques to cloud computing, resulting in robust, fault tolerant and easy to manage and operate cloud architectures. Such autonomic techniques cover evolutionary and genetic algorithms, multi-objective and combinational optimization heuristics, artificial neural networks, swarm intelligence, and multi-agents systems. Applied to Clouds, these techniques can improve how computing systems and applications are built, used, managed and optimized, maximizing the benefits for users, applications and systems by reducing the operational, maintenance and usage costs of clouds. The interplay of intelligent approaches and Clouds offers numerous challenges.

The international workshop on Intelligent Techniques and Architectures for Autonomic Clouds (ITAAC 2011) was held in association with 4th IEEE/ACM International Conference on Utility and Cloud Computing (UCC 2011)in Melbourne Australia to bring together researchers and practitioners across Cloud Computing, Intelligent Systems, and Autonomic Computing to discuss issues at the intersection of these disciplines. Key questions to be 
addressed included: How do emerging cloud architectures satisfy or contradict the vision of autonomic computing? How does the vision of autonomic computing satisfy the vision of self managing and self healing clouds? How do contemporary and emerging intelligent techniques support and enable both of these? Academics, researchers and practitioners were invited to submit original work on the theory and practice of intelligent and autonomic clouds. Papers on position statements, theoretical and industrial perspectives, and lessons learned, comparisons, evaluations and technical contributions related to intelligent autonomic clouds were invited.

Following the ITAAC workshop, the four papers described here were revised and extended and, following further peer review, accepted for this Special Issue of the Journal of Cloud Computing Advances, Systems and Applications.

1. Context Caches in the Clouds

Context information is traditionally collected from distributed digital artifacts and services and made available to similarly distributed, and often mobile, context consuming applications via context brokers or servers. Contextual data has a strong temporal element i.e. it remains valid for a period of time, and hence is an ideal candidate for caching strategies that aim to exploit such locality of reference. However, different types of contextual information have varying temporal validity durations and a varied spectrum of access frequencies as well. Such variation affects the suitability of a single caching strategy. An ideal exploitation of the caching mechanism should utilize dynamic strategies based on the type of context data, quality of service heuristics and access patterns and frequencies of context consuming applications. This paper presented an investigation of the utility of various context-caching strategies and proposed the employment of a bipartite caching mechanism in a cloud-based context broker that facilitated context provisioning between context providing services and consuming applications.

2. Software Level Green Computing For Large Scale Systems Green Computing addresses energy conservation in computer system by applying different techniques at software and hardware level. Energy efficient compiler is one of the software level green computing techniques, which contains bundles of information related to software structure and execution. The focus of this paper is identification and implementation of various green aspects for energy conservation at compiler level. A Distributed Green Compiler (DGC) was presented in this work that is hardware independent and uses an existing distributed compiler. It distributes source code of software over a network, reshapes binary code by applying green strategies during code transformation at compile time and gives green suggestion to software programmer for energy conservation.

3. An Architecture for Integrated Intelligence in Urban Management using Cloud Computing This paper advocates the application of cloud capacity to support the information, communication and decision making needs of a wide variety of stakeholders in the complex business of the management of urban and regional development. The complexity lies in the interactions and impacts embodied in the concept of the urban-ecosystem. The effective use of integrated environmental management systems is greatly enhanced and generally structured on the principle of horizontal 
integration of information across the sectoral boundaries at the local level, and on vertical integration between levels of governance. The effectiveness of an integrated environmental management system is also enhanced by the full engagement of all stakeholders in the land management process. This paper offers a user-orientated approach based on requirements for an effective management of the urbanecosystem and the potential contributions that can be supported by the cloud computing community.

4. Dot-base62x: A Compact Textual Representation of IPv6 Address for Clouds With the transition from IPv4 to IPv6, services running in Cloud computing will face problems associated with IPv6 addressing: the notation is too long (39 bytes), there are too many variants of a single IPv6 address and a potential conflict may exist with conventional http_URL notation caused by the use of the colon (:). This paper proposes a new scheme to represent an IPv6 address with a shorter, compact notation (27 bytes), without variants or conflicts with http_URL. The proposal is known as dot-base62 $\mathrm{x}$ as it is an IPv6 address with Base62x and uses the wellknown period (or dot) as a group delimiter instead of the colon. Cloud computing, as a continuously emerging mainstream of network-based applications, is likely to be a forerunner in the use of IPv6 as the base protocol. As a result, Cloud computing will benefit most from the new, compact and user-friendly textual representation of IPv6 address proposed by this paper.

Competing interests

The author(s) declare that they have no competing interests.

\section{Author details}

${ }^{1}$ School of Computing and Mathematics, Faculty of Business, Computing and Law, University of Derby, Kedleston Road, Derby DE22 1GB, UK. ${ }^{2}$ Department of Computing, Faculty of Engineering and Physical Sciences, University of Surrey, Guildford, Surrey GU2 7XH, UK.

Cite this article as: Antonopoulos et al: Intelligent techniques and architectures for autonomic clouds: introduction to the itaac special issue. Journal of Cloud Computing: Advances, Systems and Applications 2012 1:18.

Submit your manuscript to a SpringerOpen ${ }^{\circ}$ journal and benefit from:

- Convenient online submission

- Rigorous peer review

- Immediate publication on acceptance

- Open access: articles freely available online

- High visibility within the field

- Retaining the copyright to your article

Submit your next manuscript at $>$ springeropen.com 